\title{
Bypass grafting with coronary endarterectomy: Immediate and long-term results
}

\author{
Giuseppe Marinelli, MD ${ }^{\mathrm{a}}$ \\ Bruno Chiappini, MD \\ Marco Di Eusanio, MDa \\ Roberto Di Bartolomeo, MD \\ Ilaria Caldarera, MD $^{\mathrm{b}}$ \\ Cinzia Marrozzini, MD ${ }^{\mathrm{b}}$ \\ Antonio Marzocchi, MD \\ Angelo Pierangeli, $\mathrm{MD}^{\mathrm{a}}$
}

From the Departments of Cardiovascular Surgery $^{\mathrm{a}}$ and Cardiology, ${ }^{\mathrm{b}}$ Policlinico S. Orsola, University of Bologna, Bologna, Italy.

Received for publication Nov 28, 2001; revisions requested Dec 13, 2001; revisions received Feb 25, 2002; accepted for publication March 11, 2002.

Address for reprints: Bruno Chiappini, MD, Department of Cardiovascular Surgery, University of Bologna, via G. Massarenti, 9-40138 Bologna, Italy (E-mail: bruno_chiappini@hotmail.com).

J Thorac Cardiovasc Surg 2002;124:553-60

Copyright $(9) 2002$ by The American Association for Thoracic Surgery

0022-5223/2002 $\$ 35.00+0 \quad \mathbf{1 2 / 1 / 1 2 4 6 7 0}$

doi: $10.1067 / \mathrm{mtc} .2002 .124670$
Background: Increased morbidity and mortality have been associated with coronary artery bypass grafting when an adjunctive coronary endarterectomy is performed. In this study we retrospectively reviewed our experience with coronary bypass and endarterectomy to evaluate the early and late outcome and to determine the independent predictors of early mortality and morbidity of this procedure.

Methods: Between May 1989 and December 2000, 107 patients underwent myocardial revascularization with coronary endarterectomy to achieve a complete revascularization. There were 90 men and 17 women; the mean age was $64 \pm 8.1$ years. Sixty-three $(58.9 \%)$ patients had a previous myocardial infarction, and 29 $(27.1 \%)$ were operated on on an emergency basis. An ejection fraction of less than $30 \%$ was present in $9(8.4 \%)$ patients. The most frequently endarterectomized vessel was the left coronary artery (74.8\%). Follow-up information was obtained from 97 patients (100\% of the long-term survivors).

Results: There were $5(4.7 \%)$ early deaths. The 72-month survival was $91.2 \% \pm$ $4.9 \%$. Seventy-seven $(83.7 \%)$ of the survivors were symptom free, and $15(16.3 \%)$ were in Canadian Cardiovascular Society class II to III. An improvement of the ejection fraction after the operation was shown in the 97 patients who underwent echocardiographic control $(P=.03)$ and angiography. The 30.4-month patency rate of the endarteriectomized coronary arteries was $72 \% \pm 11 \%$.

Conclusion: Coronary endarterectomy is a safe and effective procedure for achieving a complete revascularization in patients with end-stage coronary disease.

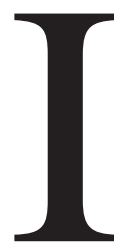

$\mathrm{n}$ the 1950s, coronary endarterectomy (CE) was introduced as a method of treating coronary artery disease $(\mathrm{CAD})^{1}$ and then was adopted by several institutions, mostly associated with coronary artery bypass brafting (CABG).

After initial reports, $\mathrm{CE}$ was questioned as an effective treatment for diffuse CAD because of increased myocardial infarction and early mortality rates $^{2}$ and was thereafter used much less frequently.

In the last 2 decades, however, increasing surgical population morbidity associated with advances in cardiac surgery and a better patient selection has prompted the resurgence of $\mathrm{CE}$. A complete revascularization showing acceptable results was then made feasible, even in patients with end-stage CAD. ${ }^{3-6}$

In our institution CE was carried out on a small group of highly selected patients when distal anastomosis to an adequately sized vessel was not possible.

The aim of this study was to assess, retrospectively, the early and late results of 
$\mathrm{CE}$ and $\mathrm{CABG}$ and to determine the independent predictors of early mortality and myocardial infarction after surgical intervention.

\section{Material and Methods Patients' Profiles}

Between May 1989 and December 2000, 107 patients underwent myocardial revascularization with $\mathrm{CE}$ at the University of Bologna to achieve a complete revascularization. There were $90(84.1 \%)$ men and $17(15.9 \%)$ women, with a mean age of $64 \pm 8.1$ years (range, 41-81 years). Fifty (46.7\%) patients had a previous history of smoking; hypertension was present in $49(45.8 \%)$ patients, and previous myocardial infarction had been experienced in 63 (58.9\%). Unstable angina was present in 54 (50.5\%) patients. Twenty-nine $(27.1 \%)$ patients were operated on as an emergency. Impairment of the ventricular function (ejection fraction [EF] $<30 \%$ ) was present in $9(8.4 \%)$ patients. Three $(2.8 \%)$ patients had undergone a previous myocardial revascularization (Table 1).

All procedures were performed with extracorporeal circulation and moderate hypothermia $\left(31^{\circ} \mathrm{C}\right)$; myocardial protection was obtained by means of intermittent antegrade crystalloid cardioplegia (St Thomas Hospital Solution) and topical cooling with cold saline $\left(4^{\circ} \mathrm{C}\right)$. An endarterectomy was performed by using a manual technique $^{7}$ and, for the most part, was performed on occluded $(65.4 \%)$ vessels in the preoperative coronary angiogram. As much plaque as possible was removed, typically between 4 and $10 \mathrm{~cm}$. If residual plaque was thought to remain in the distal vessel, the arteriotomy was enlarged as much as necessary to allow complete removal of the plaque, especially on the left anterior descending artery (LAD). The endarteriectomized vessels were flushed with heparinized solution to remove small residual particles. When a $\mathrm{CE}$ was performed on the $\mathrm{LAD}$, the internal thoracic artery was sewn to a saphenous vein patch, which was used to reconstruct the vessel in all cases. A saphenous vein graft was used as a conduit to the arteriectomy site in the other vessels.

A CE was performed in a single vessel for each patient on the arteries having a diameter of greater than $1.5 \mathrm{~mm}$; the vessels were the left coronary artery in $80(74.8 \%)$, the right coronary artery in $8(7.5 \%)$, the descending posterior artery in $8(7.5 \%)$, the obtuse marginal artery in 7 (6.5\%), and the diagonal artery in $4(3.7 \%)$ patients.

The mean extracorporeal circulation and aortic crossclamp times were $124 \pm 43$ minutes (minimum, 60 minutes; maximum, 400 minutes) and $80 \pm 25$ minutes (minimum, 28 minutes; maximum, 165 minutes), respectively. The associated procedures were aortic valve replacement in 5 (4.7\%), mitral valve replacement in $4(3.7 \%)$, left ventricular aneurysmectomy in $2(1.9 \%)$, and ascending aorta replacement in $2(1.9 \%)$ patients (Table 2).

Patients were anticoagulated postoperatively. Low-molecularweight heparin was initiated 6 hours after arrival in the intensive care unit. After 2 days, this was replaced by warfarin sodium for the following 6 months, maintaining an international normalized ratio of 2.0 to 2.5 . Then aspirin $(300 \mathrm{mg} / \mathrm{d})$ was initiated and continued indefinitely.

\section{Data Collection}

The medical records of all patients were retrospectively reviewed. The data collected included the following: (1) demographics; (2) comorbidity risk factors; (3) operative data; (4) mortality; and (5) postoperative complications defined as myocardial infarction (new Q-wave or loss of R-wave progression across the chest leads; creatine kinase $\mathrm{MB}>10 \%$ ), low cardiac output (a newly placed intra-aortic balloon pump [IABP] or the use of inotropes for $>48$ hours), malignant ventricular arrhythmias, bleeding requiring a rethoracotomy, stroke, respiratory insufficiency (mechanical ventilatory support $>48$ hours), renal failure requiring dialysis, and mediastinal infection.

\section{Follow-Up}

A questionnaire was administered to the patients included in the study investigating survival, symptoms, long-term medical management, readmissions and reinterventions, and any surgery-re- 
TABLE 1. Demographic characteristics

\begin{tabular}{|c|c|c|}
\hline Variables & Patients (n) & Patients (\%) \\
\hline \multicolumn{3}{|l|}{ Sex } \\
\hline Male & 90 & 84.1 \\
\hline Female & 17 & 15.9 \\
\hline Age (y) & \multicolumn{2}{|c|}{$64 \pm 8.1$} \\
\hline \multicolumn{3}{|c|}{ Hypertension } \\
\hline Yes & 49 & 45.8 \\
\hline No & 58 & 54.2 \\
\hline \multicolumn{3}{|l|}{ Smoking } \\
\hline Yes & 50 & 46.7 \\
\hline No & 57 & 53.3 \\
\hline \multicolumn{3}{|c|}{ Family history } \\
\hline Yes & 43 & 40.2 \\
\hline No & 64 & 59.8 \\
\hline \multicolumn{3}{|l|}{ Diabetes } \\
\hline Yes & 22 & 20.6 \\
\hline No & 85 & 79.4 \\
\hline \multicolumn{3}{|c|}{ Hyperlipidemia } \\
\hline Yes & 43 & 40.2 \\
\hline No & 64 & 59.8 \\
\hline \multicolumn{3}{|c|}{ Previous myocardial infarction } \\
\hline Yes & 63 & 58.9 \\
\hline No & 44 & 41.1 \\
\hline \multicolumn{3}{|c|}{ Previous stroke } \\
\hline Yes & 6 & 5.6 \\
\hline No & 101 & 94.4 \\
\hline \multicolumn{3}{|c|}{ Aortic valve disease } \\
\hline Yes & 6 & 5.6 \\
\hline No & 101 & 94.4 \\
\hline \multicolumn{3}{|c|}{ Mitral valve disease } \\
\hline Yes & 4 & 3.7 \\
\hline No & 103 & 96.3 \\
\hline \multicolumn{3}{|c|}{ Left ventricular aneurysm } \\
\hline Yes & 2 & 1.9 \\
\hline No & 105 & 98.1 \\
\hline \multicolumn{3}{|c|}{ Unstable angina } \\
\hline Yes & 54 & 50.5 \\
\hline No & 53 & 49.5 \\
\hline \multicolumn{3}{|l|}{$\mathrm{EF}<30 \%$} \\
\hline Yes & 9 & 8.4 \\
\hline No & 98 & 91.6 \\
\hline \multicolumn{3}{|l|}{ NYHA } \\
\hline 1 & 1 & 0.9 \\
\hline II & 33 & 30.8 \\
\hline III & 54 & 53.3 \\
\hline IV & 14 & 15 \\
\hline \multicolumn{3}{|l|}{ Status } \\
\hline Elective & 78 & 72.9 \\
\hline Urgent & 29 & 27.1 \\
\hline \multicolumn{3}{|l|}{ COPD } \\
\hline Yes & 17 & 15.9 \\
\hline No & 90 & 84.1 \\
\hline \multicolumn{3}{|c|}{ Chronic renal failure } \\
\hline Yes & 4 & 3.7 \\
\hline No & 103 & 96.3 \\
\hline \multicolumn{3}{|c|}{ Previous CABG } \\
\hline Yes & 3 & 2.8 \\
\hline No & 104 & 97.2 \\
\hline
\end{tabular}

Continuous data are expressed as mean \pm 1 SD. COPD, Chronic obstructive pulmonary disease.
TABLE 2. Operative characteristics

\begin{tabular}{lcc}
\hline Variables & Patients (n) & Patients (\%) \\
\hline AVR & & \\
$\quad$ Yes & 5 & 4.7 \\
$\quad$ No & 102 & 95.3 \\
MVR & & \\
$\quad$ Yes & 4 & 3.7 \\
$\quad$ No & 103 & 96.3 \\
Ventricular aneurysmectomy & & \\
$\quad$ Yes & 2 & 1.9 \\
$\quad$ No & 105 & 98.1 \\
CE on nonoccluded vessel & & \\
$\quad$ Yes & 70 & 65.4 \\
$\quad$ No & 37 & 34.6 \\
CPB time (min) & & $124 \pm 43$ \\
Aortic crossclamp time (min) & & $80 \pm 25$
\end{tabular}

$A V R$, Aortic valve replacement; $M V R$, mitral valve replacement; $C P B$, cardiopulmonary bypass.

lated complications. A series of 97 patients underwent echocardiographic examination and angiographic control.

\section{Statistical Analysis}

Statistical analysis was performed by using SPSS 7.0 statistical software (SPSS Inc, Chicago, Ill). Continuous variables were expressed as mean $\pm 1 \mathrm{SD}$, and categoric variables were expressed as percentages. All variables were first analyzed with univariate analysis (impaired 2-tailed $t$ test, $\chi^{2}$ test, or Fisher exact test when appropriate) to determine whether any single factor influenced hospital mortality and postoperative myocardial infarction. Variables that achieved $P$ values of less than .05 in the univariate analysis were examined with multivariate analysis by means of forward stepwise logistic regression to evaluate independent risk factors for hospital mortality and myocardial infarction after surgical intervention.

Survival data were analyzed by using the standard KaplanMayer actuarial technique for estimation of survival probabilities.

\section{Results}

\section{Early Outcome}

Five (4.7\%) patients died during hospitalization as a result of multiorgan failure $(\mathrm{n}=1)$, ventricular fibrillation $(\mathrm{n}=$ $2)$, and low cardiac output $(\mathrm{n}=2)$. Of these 5 patients, 4 were preoperatively critical and operated on as an emergency.

The first patient was a 70-year-old man operated on as a result of a severe acute ischemic mitral insufficiency and treated with CABG and mitral anuloplasty. The CE was performed on the right coronary artery supplying an already infarcted territory. Despite high inotropic support and temporary mechanical circulatory support with IABP, he died as a result of low cardiac output 3 days after the operation. The second patient was an 80-year-old man with unstable angina and preoperative renal failure requiring dialysis. A stroke occurred postoperatively, and multiorgan failure was 
TABLE 3. Postoperative results

\begin{tabular}{|c|c|c|}
\hline Variables & Patients (n) & Patients $(\%)$ \\
\hline \multicolumn{3}{|c|}{ Acute myocardial infarction } \\
\hline Yes & 2 & 1.9 \\
\hline No & 105 & 98.1 \\
\hline \multicolumn{3}{|c|}{ Hemodynamic instability } \\
\hline Yes & 10 & 9.3 \\
\hline No & 97 & 90.7 \\
\hline \multicolumn{3}{|l|}{ IABP } \\
\hline Yes & 6 & 5.6 \\
\hline No & 101 & 94.4 \\
\hline \multicolumn{3}{|c|}{ Ventricular fibrillation } \\
\hline Yes & 4 & 3.7 \\
\hline No & 103 & 96.3 \\
\hline \multicolumn{3}{|l|}{ Stroke } \\
\hline Yes & 1 & 0.9 \\
\hline No & 106 & 99.1 \\
\hline \multicolumn{3}{|c|}{ Atrial fibrillation } \\
\hline Yes & 23 & 21.5 \\
\hline No & 84 & 78.2 \\
\hline \multicolumn{3}{|c|}{ Renal failure } \\
\hline Yes & 4 & 3.7 \\
\hline No & 103 & 96.3 \\
\hline \multicolumn{3}{|c|}{ Respiratory failure } \\
\hline Yes & 2 & 1.9 \\
\hline No & 105 & 98.1 \\
\hline \multicolumn{3}{|l|}{ Bleeding } \\
\hline Yes & 2 & 1.9 \\
\hline No & 105 & 98.1 \\
\hline \multicolumn{3}{|c|}{ Mediastinal infection } \\
\hline Yes & 1 & 0.9 \\
\hline No & 106 & 99.1 \\
\hline
\end{tabular}

the cause of death. The last 2 patients had associated valve surgery (one of them had an EF of $<30 \%$ ). In these cases death was caused by postoperative ventricular fibrillation and the impossibility of completing cardiopulmonary bypass (Table 3).

On univariate analysis, the following factors had a significant influence on hospital mortality: advanced age $(P=$ $.026)$, history of smoking $(P=.032)$, chronic renal failure $(P<.001)$, unstable angina $(P=.023)$, mitral valve disease $(P<.001)$, prolonged cardiopulmonary bypass time $(P<$ $.001)$, mitral valve replacement $(P<.001)$, postoperative hemodynamic instability $(P=.016)$, postoperative acute renal failure $(P<.001)$, postoperative respiratory insufficiency $(P=.002)$, and ventricular fibrillation $(P<.001$; Table 4). Multivariate analysis revealed unstable angina (odds ratio $[\mathrm{OR}]=7.3, P=.006$ ), mitral valve replacement $(\mathrm{OR}=9.1, P=.002)$, and ventricular fibrillation $(\mathrm{OR}=$ 5.2, $P=.021)$ to be independent predictors of hospital mortality (Table 5).

Postoperative myocardial infarction occurred in $2(1.9 \%)$ patients. Postoperative hemodynamic instability was observed in $10(9.3 \%)$ patients, and $6(5.6 \%)$ patients were treated with IABP. Ventricular fibrillation occurred in 4
(3.7\%) patients, causing death in 2. The independent predictors of increased myocardial infarction rate, assessed by means of univariate analysis, were aortic valve replacement $(P=.002)$, prolonged cardiopulmonary bypass $(P=.04)$, and crossclamp times $(P=.019$; Table 6$)$. In the multivariate analysis aortic valve replacement remained the only independent predictor of myocardial infarction (Table 7). Other postoperative complications were atrial fibrillation $(21.5 \%)$, renal failure $(3.7 \%)$, acute respiratory failure $(1.9 \%)$, bleeding $(1.9 \%)$, and mediastinal infection $(0.9 \%)$.

\section{Late Outcome}

Follow-up was $95.3 \%$ complete. The overall survival curve is depicted in Figure 1. The 72-month survival was 91.2\% $\pm 4.9 \%$.

During a mean follow-up period of $38.03 \pm 24.5$ months (range, 1-124.4 months), there was a total of 5 deaths: 2 patients died from primary cardiac failure, 2 from malignancy, and 1 from a cerebrovascular problem. Seventyseven $(83.7 \%)$ patients were angina free; $15(16.3 \%)$ had angina (12 in Canadian Cardiovascular Society class 2, 1 in class 3 , and 2 in class 4$)$. Thirty-two (34.8\%) patients was classified as being in New York Heart Association (NYHA) II, $10(10.9 \%)$ in NYHA III, and $1(1.1 \%)$ in NYHA IV. Myocardial infarction occurred in $2(2.2 \%)$ patients. Ninetyseven $(100 \%)$ patients underwent echocardiographic control, which showed an EF improvement after surgical intervention $(49.8 \% \pm 8.8 \%$ vs $56.1 \% \pm 14.1 \%$ mean $\mathrm{EF}, P=$ $.03)$. They underwent angiographic control at a mean period of $30.4 \pm 16$ months after surgical intervention, and the patency rate for the endarterectomized coronary arteries was $72 \% \pm 11 \%$. The patency rate for bypass grafts to nonendarterectomized coronary arteries was $73 \% \pm 12 \%$, which is not different significantly from that of the endarterectomy grafts. According to the endarterectomized arteries and bypass grafts used, the patency rates were $75 \%$ for the LAD (internal thoracic artery graft), $90 \%$ for the RCA (saphenous vein graft), $80 \%$ for the descending posterior artery (saphenous vein graft), $60 \%$ for the obtuse marginal artery (saphenous vein graft), and 55\% for the diagonal artery (saphenous vein graft).

\section{Discussion}

The completeness of revascularization has been shown to correlate with improved early and late patient outcomes after CABG. ${ }^{8-11}$ Currently, in patients with end-stage coronary atherosclerosis, the options available include performing $\mathrm{CABG}$ to small distal vessels not performing any bypass to the diseased coronary territory or using $\mathrm{CE}$ as an adjunctive tool to $\mathrm{CABG}$ to achieve a complete revascularization. However, CE is reported as a time-consuming and challenging procedure, and its beneficial effect has been questioned because an increased risk of perioperative mortality and 
TABLE 4. Univariate analysis of hospital mortality

\begin{tabular}{|c|c|c|c|c|c|c|c|}
\hline \multirow[b]{2}{*}{ Variables } & \multicolumn{2}{|c|}{ Death } & \multirow[b]{2}{*}{$P$ value } & \multirow[b]{2}{*}{ Variables } & \multicolumn{2}{|c|}{ Death } & \multirow[b]{2}{*}{$P$ value } \\
\hline & $\mathbf{n}$ & $\%$ & & & $\mathbf{n}$ & $\%$ & \\
\hline \multicolumn{4}{|l|}{ Sex } & \multicolumn{4}{|l|}{ AVR } \\
\hline Male & 3 & 3.3 & NS & Yes & 1 & 20 & NS \\
\hline Female & 2 & 2.8 & & No & 4 & 3.9 & \\
\hline Age (y) & \multicolumn{2}{|c|}{$67.4 \pm 10.2$} & .026 & MVR & & & \\
\hline \multicolumn{3}{|l|}{ Hypertension } & & Yes & 2 & 50 & .000 \\
\hline Yes & 2 & 4 & NS & No & 3 & 2.9 & \\
\hline No & 3 & 5.1 & & Ventricular Aneurismectomy & & & \\
\hline \multicolumn{4}{|l|}{ Smoking } & Yes & - & - & NS \\
\hline Yes & 5 & 8.8 & .032 & No & 5 & 4.8 & \\
\hline No & - & - & & CE on non-occluded vessel & & & \\
\hline \multicolumn{4}{|l|}{ Family history } & Yes & 4 & 5.7 & NS \\
\hline Yes & 1 & 2.3 & NS & No & 1 & 2.7 & \\
\hline No & 4 & 6.3 & & Acute myocardial infarction & & & \\
\hline \multicolumn{4}{|l|}{ Diabetes } & Yes & - & - & NS \\
\hline Yes & 1 & 4.5 & NS & No & 5 & 4.8 & \\
\hline No & 4 & 4.7 & & Hemodynamic instability & & & \\
\hline \multicolumn{4}{|l|}{ Hyperlipidemia } & Yes & 2 & 20 & .016 \\
\hline Yes & 2 & 4.7 & NS & No & 3 & 3.1 & \\
\hline No & 3 & 4.7 & & CPB time (min) & \multicolumn{2}{|c|}{$204 \pm 132$} & .000 \\
\hline \multicolumn{4}{|l|}{ Previous myocardial infarction } & Aortic crossclamp time (min) & & +38 & NS \\
\hline Yes & 2 & 3.2 & NS & IABP & & & \\
\hline No & 3 & 6.8 & & Yes & 1 & 16.7 & NS \\
\hline Previous stroke & & & & No & 4 & 4 & \\
\hline Yes & - & - & NS & Ventricular fibrillation & & & \\
\hline No & 5 & 5 & & Yes & 2 & 50 & .000 \\
\hline Aortic valve disease & & & & No & 3 & 2.9 & \\
\hline Yes & 1 & 16.7 & NS & Stroke & & & \\
\hline No & 4 & 4 & & Yes & 1 & 100 & NS \\
\hline Mitral valve disease & & & & No & 4 & 3.8 & \\
\hline Yes & 2 & 5 & .000 & Atrial fibrillation & & & \\
\hline No & 3 & 2.9 & & Yes & 2 & 8.7 & NS \\
\hline Left ventricular aneurysm & & & & No & 3 & 3.6 & \\
\hline Yes & - & - & NS & Renal failure & & & \\
\hline No & 5 & 4.8 & & Yes & 2 & 50 & .000 \\
\hline Unstable angina & & & & No & 3 & 2.9 & \\
\hline Yes & 5 & 9.3 & .023 & Respiratory failure & & & \\
\hline No & - & - & & Yes & 1 & 50 & .002 \\
\hline $\mathrm{EF}<30 \%$ & & & & No & 4 & 3.8 & \\
\hline Yes & - & - & NS & Bleeding & & & \\
\hline No & 5 & 5.1 & & Yes & - & - & NS \\
\hline NYHA & & & & No & 5 & 4.8 & \\
\hline I & - & - & NS & Mediastinal infection & & & \\
\hline II & 一 & - & & Yes & - & - & NS \\
\hline III & 3 & 5.3 & & No & 5 & 4.7 & \\
\hline IV & 2 & 12.5 & & Continuous data are expressed & & D. NS, & $\overline{\text { ignificant; }}$ \\
\hline $\begin{array}{l}\text { Status } \\
\text { Elective }\end{array}$ & 2 & 2.6 & NS & $\begin{array}{l}\text { Continuous data are expressed } \\
\text { COPD, chronic obstructive pulmo }\end{array}$ & ease & $/ R$, aorti & replace- \\
\hline Urgent & 3 & 10.3 & IVO & ment; $M V R$, mitral valve replacen & $B, \mathrm{ca}$ & pulmone & ass. \\
\hline COPD & & & & & & & \\
\hline Yes & - & - & NS & & & & \\
\hline No & 5 & 5.6 & & & & & \\
\hline Chronic renal failure & & & & & & & \\
\hline Yes & 2 & 50 & .000 & TABLE 5. Multivariate ana & f H & ital mc & \\
\hline $\begin{array}{l}\text { No } \\
\text { Provipuc }\end{array}$ & 3 & 2.9 & & Variables & & & $P$ value \\
\hline $\begin{array}{l}\text { Previous CABG } \\
\text { Yes }\end{array}$ & — & - & NS & Unstable angina & & & .006 \\
\hline No & 5 & 4.8 & & Mitral valve replacement & & & .002 \\
\hline & & & & Postoperative VF & & & .021 \\
\hline & & & (continued) & Ventricular fibrillation. & & & \\
\hline
\end{tabular}

TABLE 4-Cont'd. 
TABLE 6. Univariate analysis of postoperative myocardial infarction

\begin{tabular}{|c|c|c|c|}
\hline \multirow[b]{2}{*}{ Variables } & \multicolumn{2}{|c|}{$\begin{array}{l}\text { Myocardial } \\
\text { infarction }\end{array}$} & \multirow[b]{2}{*}{$P$ value } \\
\hline & $\mathbf{n}$ & $\%$ & \\
\hline \multicolumn{4}{|l|}{ Sex } \\
\hline Male & 2 & 2.2 & NS \\
\hline Female & - & - & - \\
\hline Age (y) & \multirow{2}{*}{\multicolumn{2}{|c|}{67.5}} & NS \\
\hline \multicolumn{3}{|c|}{ Hypertension } & \\
\hline Yes & - & - & \multirow[t]{2}{*}{ NS } \\
\hline No & 2 & 3.4 & \\
\hline \multicolumn{4}{|l|}{ Smoking } \\
\hline Yes & - & - & \multirow[t]{2}{*}{ NS } \\
\hline No & 2 & 4 & \\
\hline \multicolumn{4}{|c|}{ Family history } \\
\hline Yes & 2 & 4.7 & \multirow[t]{2}{*}{ NS } \\
\hline No & - & - & \\
\hline \multicolumn{4}{|l|}{ Diabetes } \\
\hline Yes & - & - & \multirow[t]{2}{*}{ NS } \\
\hline No & 2 & 2.4 & \\
\hline \multicolumn{4}{|c|}{ Hyperlipidemia } \\
\hline Yes & 1 & 2.3 & \multirow[t]{2}{*}{ NS } \\
\hline No & - & - & \\
\hline \multicolumn{4}{|c|}{ Previous myocardial infarction } \\
\hline Yes & 1 & 1.6 & \multirow[t]{2}{*}{1.6} \\
\hline No & - & - & \\
\hline \multicolumn{4}{|c|}{ Previous stroke } \\
\hline Yes & - & - & \multirow[t]{2}{*}{ NS } \\
\hline No & 2 & 2 & \\
\hline Aortic valv & & & \\
\hline Yes & 1 & 16.7 & .006 \\
\hline No & 1 & 1 & \\
\hline Mitral valv & & & \\
\hline Yes & - & - & NS \\
\hline No & 2 & 1.9 & \\
\hline Left ventric & & & \\
\hline Yes & - & - & NS \\
\hline No & 2 & 1.9 & \\
\hline Unstable a & & & \\
\hline Yes & 1 & 1.9 & NS \\
\hline No & 1 & 1.9 & NS \\
\hline $\mathrm{EF}<30 \%$ & & & \\
\hline Yes & - & - & NS \\
\hline No & 2 & 2 & \\
\hline NYHA & & & \\
\hline 1 & - & - & NS \\
\hline II & 1 & 3 & \\
\hline III & 1 & 1.8 & \\
\hline IV & - & - & \\
\hline Status & & & \\
\hline Elective & - & - & NS \\
\hline Urgent & 2 & 2.6 & \\
\hline COPD & & & \\
\hline Yes & - & - & NS \\
\hline No & 2 & 2.2 & \\
\hline Chronic re & & & \\
\hline Yes & - & - & NS \\
\hline No & 2 & 1.9 & \\
\hline & & & (continued) \\
\hline
\end{tabular}

TABLE 6-Cont'd.

\begin{tabular}{|c|c|c|c|}
\hline \multirow[b]{2}{*}{ Variables } & \multicolumn{2}{|c|}{$\begin{array}{c}\text { Myocardial } \\
\text { infarction }\end{array}$} & \multirow[b]{2}{*}{$P$ value } \\
\hline & $\mathbf{n}$ & $\%$ & \\
\hline \multicolumn{4}{|l|}{ Previous CABG } \\
\hline Yes & - & - & NS \\
\hline No & 2 & 1.9 & \\
\hline \multicolumn{4}{|l|}{ AVR } \\
\hline Yes & 1 & 20 & .002 \\
\hline No & 1 & 1 & \\
\hline \multicolumn{4}{|l|}{ MVR } \\
\hline Yes & - & - & NS \\
\hline No & 2 & 1.9 & \\
\hline \multicolumn{4}{|l|}{ Ventricular aneurismectomy } \\
\hline Yes & - & - & NS \\
\hline No & 2 & 1.9 & \\
\hline \multicolumn{4}{|l|}{ CE on nonoccluded vessel } \\
\hline Yes & 1 & 1.4 & NS \\
\hline No & 1 & 2.7 & \\
\hline CPB time (min) & \multicolumn{2}{|c|}{151} & .04 \\
\hline Aortic crossclamp time (min) & \multicolumn{2}{|c|}{106} & .019 \\
\hline \multicolumn{4}{|l|}{ Acute myocardial infarction } \\
\hline Yes & - & - & - \\
\hline No & - & - & - \\
\hline \multicolumn{4}{|l|}{ Hemodynamic instability } \\
\hline Yes & 1 & 10 & .046 \\
\hline No & 1 & 1 & \\
\hline \multicolumn{4}{|l|}{ IABP } \\
\hline Yes & 1 & 16.7 & .006 \\
\hline No & 1 & 1 & \\
\hline \multicolumn{4}{|l|}{ Ventricular fibrillation } \\
\hline Yes & - & - & NS \\
\hline NO & 2 & 1.9 & \\
\hline \multicolumn{4}{|l|}{ Stroke } \\
\hline Yes & - & - & NS \\
\hline No & 2 & 1.9 & \\
\hline \multicolumn{4}{|l|}{ Atrial fibrillation } \\
\hline Yes & - & - & NS \\
\hline No & 2 & 2.4 & \\
\hline \multicolumn{4}{|l|}{ Renal failure } \\
\hline Yes & - & - & NS \\
\hline No & 2 & 1.9 & \\
\hline \multicolumn{4}{|l|}{ Respiratory failure } \\
\hline Yes & - & - & NS \\
\hline No & 2 & 1.9 & \\
\hline \multicolumn{4}{|l|}{ Bleeding } \\
\hline Yes & - & - & NS \\
\hline No & 2 & 1.9 & \\
\hline \multicolumn{4}{|l|}{ Mediastinal infection } \\
\hline Yes & - & - & NS \\
\hline No & 2 & & .006 \\
\hline
\end{tabular}

Continuous data are expressed as a mean \pm 1 SD. NS, Not significant $C O P D$, chronic obstructive pulmonary disease; $A V R$, aortic valve replacement; $M V R$, mitral valve replacement; $C P B$, cardiopulmonary bypass.AVR

morbidity often accompanies this technique, especially when performed on the LAD. ${ }^{2-5,12,13}$

Contrary to institutions ${ }^{5,6,14}$ in which CE is performed in a relatively high percentage (20\%-50\%) of cases, we limited this procedure to a small group of patients $(3.7 \%$ of the 
TABLE 7. Multivariate analysis of postoperative myocardial infarction

\begin{tabular}{lcc}
\hline Variables & OR & $\boldsymbol{P}$ value \\
\hline AVR & 5.23 & .022
\end{tabular}

$A V R$, Aortic valve replacement.

patients with CAD) with severely stenosed or totally occluded arteries having a diameter of greater than $1 \mathrm{~mm}$, in which a complete revascularization would have been otherwise impossible. There is a wide variation in the reported results of $\mathrm{CE}$ in conjunction with $\mathrm{CABG}$.

Brenowitz and colleagues ${ }^{5}$ reported a 30-day mortality of $6.3 \%$ for patients with a single endarterectomy. Livesay and colleagues $^{4}$ reported an early mortality rate of $4.4 \%$ in a group of 3369 patients in whom the right coronary artery was the most common site $(83 \%)$ requiring $\mathrm{CE}$.

In our series $5(4.7 \%)$ hospital deaths occurred. Of these, 4 patients were preoperatively critical and operated on on an emergency basis. We performed one endarterectomy for each patient. The most frequently endarterectomized vessel was the left coronary artery (74.8\%). Initially, endarterectomy of the right system was preferred because of its technical simplicity and a lower risk. Brenowitz and colleagues ${ }^{5}$ reported a mortality rate of $4.8 \%$ for the isolated right $\mathrm{CE}$ and $7.9 \%$ for the LAD. Livesay and coworkers ${ }^{4}$ reported a significantly increased early mortality rate $(8.5 \%)$ when $\mathrm{CE}$ was performed on the LAD, as compared with endarterectomy of vessels other than the LAD. In our experience no hospital deaths or postoperative myocardial infarctions occurred in this group of patients.

Using multivariate analysis, mitral valve replacement $(P=.002)$ and unstable angina $(P=.006)$ were the most important independent predictors of early mortality, probably reflecting the critical status of these patients and the necessity for prolonged cardiopulmonary bypass and aortic crossclamp times. In this series the mean cardiopulmonary bypass and aortic crossclamp times were $124 \pm 43$ and $80 \pm 25$ minutes, respectively.

Myocardial infarction resulting from thrombosis or residual obstruction of the vessel is reported as the main cause of postoperative morbidity and mortality. In the literature the postoperative myocardial infarction rate ranges from 5\% to $25 \%$, with mortality rates of $0 \%$ to $15 \% . .^{8-12}$ In our experience 2 postoperative myocardial infarctions occurred, and the rate was $1.9 \%$. Multivariate analysis revealed aortic valve replacement $(P=.02)$ as an independent predictor of postoperative infarction. Interestingly, performing a $\mathrm{CE}$ on a nonoccluded vessel was not indicated as a risk factor for a myocardial infarction.

Continuous data are expressed as a mean $\pm 1 \mathrm{SD}$. NS, Not significant; $C O P D$, chronic obstructive pulmonary disease; $A V R$, aortic valve replacement; $M V R$, mitral valve replacement; $C P B$, cardiopulmonary bypass. The 6-year actuarial survival was $91.2 \% \pm 4.9 \%$. This compares favorably with the rate in other studies, typically ranging from $75 \%$ and $90 \% .4,5,15$

Clinically, $83.7 \%$ of our survivors were angina free at an average interval of $33.4 \pm 20.2$ months after surgical intervention. An improvement of myocardial contractility after surgical intervention was also shown by means of echocardiographic control. At angiography, the patency rate of the grafts anastomosed to the endarterectomized vessels was $72 \% \pm 11 \%$ at a mean follow-up period of $30.4 \pm 16$ months and is similar to that seen in previously published studies. ${ }^{13-15}$ In conclusion, our data show that CE is a safe and effective procedure for a complete revascularization in patients with end-stage CAD and shows acceptable early and late results.

\section{References}

1. Bailey CP, May A, Lemmon WM. Survival after coronary endarterectomy in man. JAMA. 1957;164:641-6.

2. Effer DB, Groves LK, Sones FM, Shirley EK. Endarterectomy in the treatment of coronary artery disease. J Thorac Cardiovasc Surg. 1964;47:98-102.

3. Loop FD. Resurgence of coronary endarterectomy. J Am Coll Cardiol. 1988;11:712-3

4. Livesey JJ, Cooley DA, Hallman GL, et al. Early and late results of coronary endarterectomy. J Thorac Cardiovasc Surg. 1986;92:649-60.

5. Brenowitz JB, Kayser KL, Johnson ND. Results of coronary endarterectomy and reconstruction. J Thorac Cardiovasc Surg. 1988;95:110.

6. Christakis GT, Roo V, Frames SE, Chon E, Naylor CD, Goldman BS Does coronary endarterectomy adversely effect results of bypass surgery? J Card Surg. 1993;8:72-8.

7. Cheanvechai C, Groves LK, Reyes EA, Shirley EK, Sones FM. Manual coronary endarterectomy. J Thorac Cardiovasc Surg. 1975; 70:524-7.

8. Jones EL, Craver JM, Guyton RA, Bone DK, Hatcher CR, Riechwald $\mathrm{N}$. Importance of complete revascularization in performance of the coronary bypass operations. Am J Cardiol. 1983;51:7-12.

9. Bell MR, Gersh BJ, Scaff HV, et al. Effect of completeness or revascularization on long-term outcome of patients with three vessel disease undergoing coronary artery bypass surgery: a report from the Coronary Artery Surgery Study (CASS) Registry. Circulation. 1992; 86:446-57.

10. Jones EL, Weintraub S. Importance of completeness or revascularization during long-term follow-up after coronary artery operations. J Thorac Cardiovasc Surg. 1996;112:227-37.

11. Cukingnan RA, Carey JS, Witting JH, Brown BG. Influence of complete coronary revascularization on relief of angina. $J$ Thorac Cardiovasc Surg. 1980;79:188-93.

12. Keon WJ. Manual coronary endarterectomy and revascularization. Improving techniques and results. Ann Thorac Surg. 1981;32:427-8.

13. Minale C, Nikol S, Zander M, Uebis R, Effert S, Messmer BJ. Controversial aspects of coronary endarterectomy. Ann Thorac Surg. 1989:48:235-41.

14. Gill IS, Beanlands DS, Boyd WD, Finlay S, Keon WJ. Left anterior descending endarterectomy and internal thoracic artery bypass for diffuse coronary disease. Ann Thorac Surg. 1998;65:9- 62.

15. Djalilian AR, Shumway SJ. Adjunctive coronary andarterectomy: improved safety in modern cardiac surgery. Ann Thorac Surg. 1995; 60:1749-54 
Appendix.

Factors assessed for hospital mortality and postoperative myocardial infarction

- Sex

- Age

- Hypertension

- Smoking

- Family history

- Diabetes

- Hyperlipidemia

- Previous myocardial infarction

- Previous stroke

- Aortic valve disease

- Mitral valve disease

- Left ventricular aneurysm

- Unstable angina

- $\mathrm{EF}<30 \%$

- NYHA class

- Status
- Chronic obstructive pulmonary disease

- Chronic renal failure

- Previous CABG

- Aortic valve replacement

- Mitral valve replacement

- Ventricular aneurismectomy

- CE on nonoccluded vessel

- Cardiopulmonary bypass time

- Aortic crossclamp time

- Acute myocardial infarction

- Hemodynamic instability

- IABP

- Ventricular fibrillation

- Stroke

- Atrial fibrillation

- Renal failure

- Respiratory failure

- Bleeding

- Mediastinal infection 